\title{
New Modeling of Reflection Interference Contrast Microscopy Including Polarization and Numerical Aperture Effects: Application to Nanometric Distances Measurement and Object Profile Reconstruction
}

\author{
O. Theodoly, Z.-H. Huang, M.-P. Valignat ${ }^{*}$
}

Laboratoire Adhésion et Inflammation, INSERM U600, CNRS UMR 6212, Université de la Méditerranée, 163 Avenue de Luminy, Marseille F-13009, France

*Corresponding author. Tel: +33 (0)4 918288 67; Fax: +33 (0)4 91828851.

E-mail: marie-pierre.valignat@inserm.fr

\begin{abstract}
We have developed a new and improved optical model of reflection interference contrast microscopy (RICM) to determine with a precision of a few nanometers the absolute thickness $h$ of thin films on a flat surface in immersed conditions. The model takes into account multiple reflections between a planar surface and a multistratified object, finite aperture illumination (INA), and, for the first time, the polarization of light. RICM intensity $I$ is typically oscillating with $h$. We introduce a new normalization procedure that uses the intensity extrema of the same oscillation order for both experimental and theoretical intensity values and permits us to avoid significant error in the absolute height determination, especially at high INA. We also show how the problem of solution degeneracy can be solved by taking pictures at two different INA values. The model is applied to filled polystyrene beads and giant unilamellar vesicles of radius 10 - $40 \mu \mathrm{m}$ setting on a glass substrate. The RICM profiles $I(h)$ can be fitted for up to $2-3$ oscillation orders an,d extrema positions are correct for up to 5-7 oscillation orders. The precision of the absolute distance and of the shape of objects near a substrate is about $5 \mathrm{~nm}$ in a range from 0 to $500 \mathrm{~nm}$, even under large numerical aperture conditions. The method is especially valuable for dynamic RICM experiments and with living cells where large illumination apertures are required.
\end{abstract}

\section{Introduction}

Cell adhesion is involved in most physiological cell functions, including survival, proliferation, differentiation, migration, and activation, as well as pathological situations. Cell adhesion is a very complex phenomenon, and 
biophysicists have often approached the problem by studying simplified experimental systems involving model solid substrates and/or giant vesicles in place of cells ${ }^{1,2,3,4}$. In terms of analysis tools, reflection interference contrast microscopy (RICM) has proven to be very useful in imaging the adhesion zone between a cell or a vesicle and a flat substrate $5,6,7,8$, 9,10,11 whereas fluorescence microscopy, including total internal reflection microscopy (TIRF) ${ }^{12,13}$, has yielded information at the molecular level (e.g., the position of focal adhesion points). ${ }^{14}$ There is growing interest in obtaining quantitative information on cell-substrate interactions. For instance, the absolute distance between a cell and a substrate during approach and adhesion processes is important information in the study of adhesion mechanisms and is technically challenging to obtain. RICM is the method of choice for measuring substrate-object distances in the nanometer range. However, the accuracy of distance determination by RICM depends on the relevance of the optical model linking the intensity on RICM images with an actual object-substrate distance and numerous models have already been developed. $6,8,9,15,16$

RICM consists of imaging the contact region with a high numerical aperture antiflex objective in epi-illumination mode. The interference of the light beams reflected by the substrate and by the object implies that the detected intensity is linked to the substrate-object distance. A simple model, often used in the literature, ${ }^{17,18,19,20}$ considers only normal illumination and reflection on two interfaces (the substrate/solution and the solution/object), which are assumed to be parallel. This simple model has the advantage of allowing a straightforward distance determination via an arccosine transformation. However, the accuracy of distance determination using this simple model is subject to caution. The model has been tested by Wiegand et al, ${ }^{9}$ and their conclusion was that performing measurements with a minimum aperture allows for the determination of the relative height with an accuracy of about $2 \mathrm{~nm}$ and that absolute heights were available within the first $100 \mathrm{~nm}$ with an error of about $5 \mathrm{~nm}$. It has also been realized that taking into account only two reflecting interfaces and assuming the parallelism of interfaces cannot accurately model complex and curved objects such as vesicles and cells. The problem of multiple reflection effects have been first taken into account by Wiegand et al. ${ }^{15}$. More recently, Limozin et al. ${ }^{16}$ have also proposed an improved version of the simple arccosine model by considering three reflection planes in their model in order to take into account the reflection of the inner and outer sides of vesicle membranes. Gingell et al. ${ }^{6}$, Raedler et al. ${ }^{8}$ and Wiegand et al. ${ }^{9}$ have generalized the simple model to the case of finite illumination aperture INA. Indeed, zero-angle incidence is a poor approximation of finite aperture microscopic interferometry such as RICM, where light strikes objects at large angles of incidence. ${ }^{6}$ These models demonstrate that finite INA results in a dampening of the intensity oscillations with the substrate-object distance, which is observed experimentally. Wiegand et al. ${ }^{9}$ have also 
developed a theory that takes into account not only an illumination cone but also, for the first time, reflection at nonplanar interfaces with different slopes. This model is a substantial improvement of the RICM experiments' description and has allowed one to fit precisely RICM images of filled spherical beads close to a flat surface. However, the sophistication of the model renders its use difficult and even impossible for objects of unknown shape. This may explain why there is yet no application of this model to systems more complex than a filled spherical bead or a liquid contact zone. In the end, despite all of these recent and important improvements to RICM modeling, some optical aspects have still been overlooked, such as the effect of light polarization. Taking into account the polarization of light in RICM modeling is indeed a legitimate approach because the RICM technique is based on the use of a polarizer, a $\lambda / 4$ plate, and crossed analyzers.

Herein, we describe a new optical model for RICM that takes into account (1) multiple reflections on multiple planar surfaces, (2) finite aperture illumination, and (3) for the first time, the polarization of light. We neglect in this model only the effect of the reflection on nonparallel interfaces. Although the latter approximation implies that our model is not, strictly speaking, absolute, we demonstrate that the range of applicability of the model is by far wide enough for typical use with adhering vesicles and cells. However, the advantage of making this assumption is that our model is simple and readily applicable to any complex multilayered system, such as adhering cells or vesicles on planar substrates. To the credit of this assumption, one can also note that the complete calculation for nonplanar interfaces requires a knowledge of the object profile and is therefore not applicable to cells or deflated vesicles of unknown shape. We show here how our model permits us to reconstruct the profiles of objects with unknown shapes around the contact zone in the range 0-500 $\mathrm{nm}$. We also present in this article a new method for the normalization of intensity that permits us to compare experimental and calculated intensity profiles normalized using the intensity extrema of the same oscillation order. We apply our model and normalization method to two well-controlled experimental systems of spherical polystyrene beads and of giant phospholipid vesicles sitting on a glass substrate and show that it permits the absolute height determination with a precision around $5 \mathrm{~nm}$, even at a high numerical aperture. This makes our approach especially suitable for RICM measurement with living cells and for fast dynamics applications.

\section{Experimental Section}

2.1. Microspheres. Polybead® Polystyrene beads were obtaines from Polysciences (Warrington, PA, USA). They have a refractive index of $n_{P S}=1.55$ and a diameter $d_{\text {beads }}=74.1 \mu \mathrm{m}$ with a standard deviation of $7.78 \mu \mathrm{m}$. The stock solution at concentration $C=2.75 \%$ wt was diluted with pure water at $C=0.01 \%$ wt. 
2.2. Giant Lipid Vesicles on Glass Substrates. Giant unilamellar DOPC vesicles were prepared by electro-swelling ${ }^{21}$ in aqueous solution of sucrose at $200 \mathrm{mM}$ and $600 \mathrm{mM}$. $100 \mu \mathrm{l}$ of a freshly prepared vesicles suspension was diluted in 1 $\mathrm{ml}$ of an aqueous solution of glucose at respectively $200 \mathrm{mM}$ and $600 \mathrm{mM}$. In order to modulate the average distance between vesicles and substrates, aliquots of $\mathrm{NaCl} 300 \mathrm{mM}$ solution are added in the vesicle suspension. The vesicles suspensions were deposited in a $16 \mathrm{~mm}$-diameter cylindrical chamber with a glass cover-slide at the bottom. Before each experiment, a new thickness corrected glass cover-slides (Assistant, Karl Hecht, Sondheim, Germany) was cleaned in a Decon 90 (Decon, Hove, UK) solution at $15 \%$ wt in water, thoroughly rinsed with ultra-pure (Millipore) water and finally dried with ultra pure Nitrogen. The vesicles radius is measured by visualizing the largest cross section of the vesicles by phase contrast microscopy. The refractive index and thickness of the membrane are respectively of 1.486 and $5 \mathrm{~nm}^{8}$.

2.3. RICM Measurements. We used a Zeiss axiovert 200 inverted microscope (Carl Zeiss, Jena, Germany) equipped with a Zeiss Neofluar 63/1.25 antiflex objective, a crossed polarizers cube and a C7780 camera (Hamamatsu, Tokyo, Japan), and adjustable field and aperture stops. The illumination numerical aperture INA is determined for each given field aperture opening by measuring the diameter of the illumination cone on a screen positioned at two different heights. The smallest available illumination numerical aperture is INA=0.48. The source was an X-cite 120Q lamp (Exfo, Mississauga, Canada) coupled to a narrow bandpass filter $(\lambda=546 \mathrm{~nm} \pm 12 \mathrm{~nm})$. The exposure time was of $40 \mathrm{~ms}$ and $300 \mathrm{~ms}$ for INA of respectively 1.2 and 0.48. A typical RICM image of settling bead or adhering vesicle (Figure 1) presents a central disk, which corresponds to the contact zone, surrounded by Newton rings which correspond to the interference pattern between the substrate and the object. The raw intensity $I$ was obtained by averaging radially the signal in the rings. The normalized intensity $I_{N}$ is obtained via Eq.1:

Eq. 1

$$
I_{N}=\frac{\left(I-I_{\min }\right)}{\left(I_{\max }-I_{\min }\right)}
$$

where $I_{\max }$ and $I_{\min }$ correspond to the experimental maximum and minimum intensity in the interference fringes pattern. This normalization process has the advantage to eliminate background noise contribution.

2.4. Modeling of Object/ Substrate Interface. For RICM image reconstruction, the reflecting surfaces of the object and the substrate are taken to be locally parallel. The curved object surface is assumed to consist of a succession of infinitesimal horizontal steps (Figure 2-a). Interference occurs between light reflected at the glass/solution interface, the solution/object interface, and eventually at interfaces inside the object (e.g. in the case of vesicles the added interface is due to the existence of a membrane of finite thickness). Below the glass, immersion oil matches the indices of refraction of the 
glass and the objective lens, preventing reflection at the lower interface. The substrate/solution/object system is modeled by a multilayer of parallel dielectric layers indexed by $j$ and characterized by a refractive index $n_{j}$ and a thickness $d_{j}$ (Figure 2$\mathrm{b}$ and c). The refractive index are taken equal to $n_{0}=1.525$ for the glass substrate, $n_{1}=1.334$ for water and $n_{1}=1.339$ for glucose solution, $n_{2}=1.486$ and 1.550 for the vesicle membrane and the polystyrene bead, and $n_{3}=1.346$ for the sucrose solution. The thickness of the lipid layer is of $5 \mathrm{~nm}^{8}$ and the thickness $h=d_{l}$ of the solution layer between the substrate and the object is a variable in the model that depends on the shape of he object.

\section{Results and Discussion}

The aim of this article is to demonstrate the potential use of a new optical model to measure nanometric distances precisely using RICM (e.g. thin film thickness and object-substrate distances). We first introduce our optical model of RICM and our method for intensity normalization. We then present experimental applications with two optically wellcontrolled systems. First, calibrated polystyrene beads are used to validate quantitatively the model accuracy with RICM images taken under several optical conditions. Second, giant phospholipid vesicles adhering to a glass surface are used to show how the model permits us to treat data with more complex objects. We will determine the absolute height of vesicles and demonstrate how a picture taken at different INA values permit us to solve the degeneracy of the solutions. Finally, we will explain how to reconstruct the topography of objects of unknown shape. Beads and vesicles are obviously very simplified models for living cells, but their optical properties are readily accessible whereas cells are characterized by highly complex structures on their outsides (extracellular matrix), in their membranes (high concentration of diverse proteins), and in their insides (organelles and nucleus). Beads and vesicles are therefore more suitable than cells in validating an optical model. However, we will show that the model yields a significant improvement to data taken at a large numerical aperture, INA. It is therefore particularly adapted for RICM experimentation in which large INA conditions are required to avoid the perturbation of images by reflection from the inner organelles.

3.1 RICM Modeling. 3.1.1. Modeling of the Reflected Intensity. RICM observations are made in monochromatic epiillumination with a crossed polarizer/analyzer cube and an antiflex objective (Figure 1). The light first passes through a polarizer, crosses a quarter-wavelength plate and the objective lens twice (before and after reflection on the sample), and finally passes through the analyzer. The quarter wavelength plate orientation is set at $45^{\circ}$ with respect to the orientation of the polarizer. The combination of crossed polarized and quarter-wavelength plate suppresses all stray light that retains its original sense of polarization, which permits us to increase the contrast of the image. 
Our RICM modeling is inspired from Jones matrix calculations developed for ellipsometry. ${ }^{22,23,24}$ An uniform monochromatic plane wave passes through the RICM setup. The cone of light impinging the sample integrates contributions from all beams with angle of incidence $\theta$ ranging from 0 to $\theta_{l}$ and azimuth $\varphi$ ranging from 0 to $2 \pi$ (Figure 3.a). The numerical illumination aperture is defined as $I N A=n_{1} \sin \theta_{1}$. Let us first consider a single incidence $\theta$ and a given incident plane of azimuth $\varphi$. The sample is characterized by its reflection coefficients parallel $\left(R_{p}\right)$ and perpendicular $\left(R_{s}\right)$ to the plane of incidence. Following Azzam and Bashara's sign conventions, ${ }^{22}$ the frame attached to the incident beam is $(\vec{p}, \vec{s}, \vec{k})$ where $\vec{p}$ and $\vec{s}$ are respectively the direction parallel and perpendicular to the incident plane and $\vec{k}$ is the wave vector. Without a loss of generality, we define $\varphi$ as the angle between $\vec{p}$ and the fixed direction of the polarizer. For each beam with incidence $\theta$ on the reflective surface, the orientations of the polarizer, the quarter-wave plate and the analyzer around the beam axis are respectively specified by azimuthal angles $\varphi,(\varphi+\pi / 4),(\pi-(\varphi+\pi / 4)),(\pi / 2-\varphi)$ as depicted on Figure 3.b. All azimuthal angles are taken to be positive in a counterclockwise sense, looking into the beam from the $\vec{p}$ direction of the optical system. Let $A_{0}$ be the amplitude of the incident light and consider $\vec{k}$ above the objective lens in order to confine the calculation to two dimensions. The components of the Jones vector emerging from the analyzer can be expressed as

Eq. $2 \quad \mathbf{E}_{A}=\mathbf{T}_{A} \mathbf{R}(-\pi / 4) \mathbf{T}_{\lambda / 4} \mathbf{R}(3 \pi / 4-\varphi) \mathbf{T} \mathbf{R}(-\varphi-\pi / 4) \mathbf{T}_{\lambda / 4} \mathbf{R}(\pi / 4)\left(\begin{array}{c}\sqrt{2} / 2 \\ 0\end{array}\right) A_{0}$

where $\mathbf{R}_{\mathrm{X}}$ represents the rotational matrix (with rotation angle $X$ ) and $\mathbf{T}_{\mathrm{A}}, \mathbf{T}_{\mathrm{s}}$, and $\mathbf{T}_{\lambda / 4}$ are the Jones matrices for the analyzer, the specimen, and the quarter-wave plate. $\mathbf{R}_{\mathrm{X}}, \mathbf{T}_{\mathrm{A}}, \mathbf{T}_{\mathrm{s}}$, and $\mathbf{T}_{\lambda / 4}$ are equal to

Eq. 3

$$
\mathbf{T}_{A}=\left(\begin{array}{ll}
1 & 0 \\
0 & 0
\end{array}\right), \quad \mathbf{T}_{s}=\left(\begin{array}{cc}
R_{p} & 0 \\
0 & R_{s}
\end{array}\right), \quad \mathbf{T}_{\lambda / 4}=\left(\begin{array}{cc}
1 & 0 \\
0 & e^{i \pi / 2}
\end{array}\right)
$$

and

Eq. 4

$$
\mathbf{R}_{X}=\left(\begin{array}{cc}
\cos X & -\sin X \\
\sin X & \cos X
\end{array}\right)
$$


The evaluation of the matrix sequence leads to

Eq. 5

$$
\mathbf{E}_{A}=\frac{A_{0} \sqrt{2}}{4}\left(R_{s}-R_{p}\right) \mathbf{u}_{A}
$$

The value of the emerging electric field is independent of the azimuth angle $\varphi$ of the plane of incidence but dependent on the incident angle $\theta$ through the reflection coefficients. Using eq. 5, one can find the total reflected intensity $I_{\theta}$, for a cone of light with incidence $\theta$

Eq. 6

$$
I_{\theta}=\Sigma \int_{0}^{2 \pi} \mathbf{E}_{A}^{*} \mathbf{E}_{A} d \varphi=\frac{A_{0}^{2}}{4} \pi \Sigma\left|R_{s}-R_{p}\right|^{2}=\alpha\left|R_{s}-R_{p}\right|^{2}
$$

where $\Sigma$ is a constant characteristic of the microscope geometry and $\alpha=\frac{A_{0}^{2}}{4} \pi \Sigma$ is a constant independent of the optical properties of the sample.

The total reflected flux $I$ under the convergent illumination conditions of a microscope is obtained by the integration of eq. 6 over the incident light cone as

Eq. 7

$$
I=\frac{1}{1-\cos \theta_{\max }} \int_{0}^{\theta_{1}} I_{\theta} \sin \theta d \theta=\frac{\alpha}{1-\cos \theta_{\max }} \int_{0}^{\theta_{1}}\left|R_{s}-R_{p}\right|^{2} \sin \theta d \theta
$$

3.1.2. Modeling of a Multilayer Object. The multilayer approach, developed by F. Abelès ${ }^{25}$ and later by Azzam and Bashara $^{22}$ for ellipsometry is a $2 \times 2$ matrix method of calculating reflection coefficients on objects composed of multiple flat and homogeneous layers (Figure 2). A matrix $\mathbf{M}_{\mathrm{j}}$ is associated with each layer $j$ of the multilayer

Eq. 8

$$
\begin{gathered}
\mathbf{M}_{j}=\left(\begin{array}{cc}
\cos \beta_{j} & \frac{i \sin \beta_{j}}{N_{j}} \\
i N_{j} \sin \beta_{j} & \cos \beta_{j}
\end{array}\right) \\
\beta_{j}=\frac{2 \pi d_{j}}{\lambda} n_{j} \cos \theta_{j}
\end{gathered}
$$

Eq. 9 
Eq. 10

$$
N_{j}=n_{j} \cos \theta_{j} \text { for } s \text {-polarization and } N_{j}=\frac{n_{j}}{\cos \theta_{j}} \text { for } p \text {-polarisation }
$$

Matrix $\mathbf{M}_{j}$ permits us to link the electric and magnetic components in the layers $(j-1)$ and $(j)$ at the interface between layers $(j-1)$ and $(j)$

Eq. 11

$$
\left(\begin{array}{l}
E_{j-1} \\
B_{j-1}
\end{array}\right)_{z=d_{j-1}}=\mathbf{M}_{j}\left(\begin{array}{l}
E_{j} \\
B_{j}
\end{array}\right)_{z=d_{j-1}}
$$

The matrix $\mathbf{M}$ of the medium formed of $m$ layers is finally:

Eq. 12

$$
\mathbf{M}=\prod_{j=1}^{m} \mathbf{M}_{j}=\left(\begin{array}{ll}
m_{11} & m_{12} \\
m_{21} & m_{22}
\end{array}\right)
$$

Eq.

Equation 11 links the field components of the 0/1 interface contiguous with the glass slide to the field components at the $m / m+1$ interface contiguous with the outer medium. The total field in the medium 0 results from the superposition of an incident $\left(\mathrm{E}_{0}^{+}\right)$and a reflected $\left(\mathrm{E}_{0}^{-}\right)$field. By definition, the reflection coefficient of the object sandwiched between semiinfinite media $(0)$ and $(\mathrm{m}+1)$ is:

Eq. 13

$$
r_{0 m+1}=\frac{E_{0}^{-}}{E_{0}^{+}}
$$

which can be expressed in term of the matrix coefficients as:

Eq. 14

$$
r_{0 m+1}=\frac{m_{11} N_{0}-m_{22} N_{m+1}+m_{12} N_{0} N_{m+1}-m_{21}}{m_{11} N_{0}+m_{22} N_{m+1}+m_{12} N_{0} N_{m+1}+m_{21}}
$$

Therefore, reflection coefficients $R_{p}$ and $R_{s}$ parallel and perpendicular, respectively, to the plane of incidence of a multifilms structure (film $1,2, \ldots, m)$ sandwiched between semi-infinite ambient media $(0)$ and substrate media $(m+1)$ are

Eq. 15

$$
R_{p}=r_{0 m+1} \quad \text { with all efficient optical index of the form } N_{j}=\frac{n_{j}}{\cos \theta_{j}}
$$


3.2. Validation of the Model with Respect to Model Spherical Objects. An object sitting close to a substrate presents a characteristic fringe pattern in RICM, where a fringe corresponds to a constant distance between the substrate and the object surface (Figure 1). The fringe pattern with spherical objects is made of circular concentric Newton rings. In the following text, the central area corresponding to the smallest distance between an object and a substrate is called the contact zone even if the object is not in physical contact. The typical range for the object-substrate distance equilibrium is on the order of 0 to $200 \mathrm{~nm}$ in the examples considered here. The experimental intensity profiles $I(x)$ are recorded versus the radial distance $x$ from the center of the contact zone in the plane of the substrate, whereas intensity calculations $I_{c}(h)$ are performed versus the vertical distance $h$ between the substrate and the object. The variable transformation from $h$ to $x$ for spherical objects of diameter $R$ and a minimum separation distance $h_{c}$ can be expressed as

$$
h(x)=h_{c}+R-\sqrt{R^{2}-x^{2}}
$$

3.2.1. Effect of INA on Calculated Intensities. To study dynamical processes such as the height dynamic fluctuation of the vesicle membrane, it may be interesting to increase the frame rate acquisition. In order to compensate for the shortened exposure time, it is interesting to maximize the illumination intensity by increasing the illumination numerical aperture, INA. Also, for experiments with living cells, it is interesting to work at large INA to limit the intensity reflected by inner organelles. Models of RICM considering normal incidence are inadequate for these applications at large INA. Examples of our theoretical calculations taking into account INA are presented in Figure 4 for a vesicle on a glass substrate. We consider a DOPC membrane of thickness $5 \mathrm{~nm}$, a sucrose solution inside the vesicle, and a glucose solution outside the vesicle. The theoretical intensity normalized by the first minimum and maximum intensities are reported versus the substrate-vesicle distance $h$ for several INA values. The intensity follows an oscillating function with $h$. The first minimum of intensity corresponds to height $h_{1}$ different from zero, whereas for a filled object the first minimum of intensity corresponds to the object in contact with a substrate (i.e., a height equal to zero). This shift from zero to $h_{l}$ is due to the reflection of light on the inner membrane interface. ${ }^{15,16}$ Interestingly, Figure 4 shows that the position of the first minimum is almost independent of the INA whereas all other extrema are shifted to larger values for larger INAs ${ }^{8,13}$ One can also note that the amplitude of the oscillations are significantly dumped as the INA is increased, especially for INA larger than 
0.8. The lowest minimum and largest maximum always correspond to, respectively, the first minimum and first maximum. However, all other extrema values depend strongly on the oscillation order and the numerical aperture value.

3.2.2.Intensity Normalization Procedure. The results of Figure 4 also suggest that the normalization procedure of experimental and theoretical data using the intensity extrema can be a source of error. Indeed, the intensity extrema of different orders are not equivalent. Hence, for a given experiments, one does not know a priori to which oscillation order the minimum and maximum intensities recorded belong to because the object-substrate distance $h_{c}$ is a priori unknown. After the normalization of experimental data with experimental $\mathrm{I}_{\min }$ and $\mathrm{I}_{\max }$, one would like to make a comparison with calculated data using a model. To make this comparison, the calculated data also have to be normalized using the intensity extrema corresponding to the same oscillation orders as experimental $\mathrm{I}_{\min }$ and $\mathrm{I}_{\max }$. An experimental intensity profile $I(x)$ versus radial distance $x$ is always oscillatory, and the first question that arises is, to which oscillation order does the first experimental oscillation corresponds? The specific problem of the degeneracy of the solution can in principle be solved by taking pictures at two different wavelengths for the incident light, ${ }^{26}$ and we show later in this article that an alternative method consists of taking pictures under two different INA conditions. Let us focus here on the specific problems of the normalization procedure and assume first that the objects are in close contact (i.e., that the distance ranges between 0 and $100 \mathrm{~nm}$ ), which is often assumed ${ }^{27}$ for vesicles or cells settled on a glass substrate. One can then consider that the minimum object-substrate distance $h_{c}$ is smaller than the distance $h_{M}$ corresponding to the first theoretical maximum in Figure 5-a. Therefore, the localization of the maximum is unambiguous but the minimum has to be determined with caution and depends on INA and $h_{c}$.

Let us use the theoretical curve $I(h)$ of Figure 5-a, calculated for a vesicle on a glass substrate, to predict the different types of experimental curves $I(x)$ (cf. Figure 5-b) that can be encountered depending on the unknown value $h_{c}$. The theoretical curve $I(h)$ of a vesicle on a glass substrate is divided in three regions. Region I corresponds to $h<h_{l}$, where $h_{l}$ is the distance corresponding to the first-order intensity minimum. In region I, the intensity decreases with increasing $h$. Therefore, if $h_{c}$ belongs to region I, then intensity $I(x)$ will first decrease with $x$ from the origin $x=0$ taken at the center of the contact zone. The contact zone is surrounded by a dark ring (Figure 5.c, red insert). Region II is delimited by $h_{l}$ and $h_{i}$, where $h_{i}$ corresponds to the smallest thickness above $h_{l}$ for which the intensity is equal to the intensity of the second minimum. If $h_{c}$ belongs to region II, then the minimum intensity on the experimental intensity profile is at $x=0$. Hence, after normalization using eq. 1 , the normalized intensity at $x=0$ is null. The contact zone is surrounded by a bright ring (Figure 5.c yellow insert). Finally, region III is limited by $h_{i}$ and $h_{M}$. If $h_{c}$ belongs to region III, the minimum experimental 
intensity corresponds to the second-order minimum in $h_{2}$. The $I(x)$ profiles after normalization are not null in $x=0$, and the intensity first increases with $x$ from $x=0$. The contact zone is surrounded by a bright ring (Figure 5.c green insert). Normalized calculated profile $I(x)$ and RICM images for DOPC vesicles on a glass substrate are presented in Figure 5.b for $h_{c}$ belonging to region I, II or III. A detailed quantitative comparison of our model combined with our normalization with the simple arccosine model is presented in Supporting Information (Figures S1 and S2).

The normalization of the experimental intensity profiles $I(x)$ is made versus the maximal and minimal experimental intensities found in the interference fringe pattern. Hence, the minimum intensity used for the normalization of the experimental profiles $I(x)$ depends on which region (I, II or III) $h_{c}$ belongs to. $I_{\min }$ used for normalization corresponds to $I\left(h_{1}\right)$ if $h_{c}$ belongs to region I, to $I\left(h_{c}\right)$ if $h_{c}$ belongs to region II and to $I\left(h_{2}\right)$ if $h_{c}$ belongs to region III. To fit the experimental data, it is crucial that the same normalization procedure is applied to the calculated intensity profiles $I(x)$. Hence, in our normalization procedure of calculated intensities, we consider only calculated intensities for $h \geq h_{c}$ for the determination of the $I_{\min }$ and $I_{\max }$ values used in (eq. 1).

3.2.3. Filled Beads. Figure 6 presents the RICM pictures and intensity profiles $I(x)$ measured for the same polystyrene bead on a glass substrate at three different illumination numerical apertures INA. The INAs corresponding to the three images have been measured at $0.48,0.98$ and 1.2. Several calculations using different models are also plotted in Figure 6. We present the profiles calculated with our model for all INA conditions with the same bead-substrate distance $h_{c}$ of $22 \mathrm{~nm}$ and no other fitting parameter. We have also reported on Figure 6 the intensity profiles calculated using the model of Wiegand et al. ${ }^{9}$ that takes into account nonplanar interfaces effects. Finally, we also report the result of a calculation based on our computation procedure, but using Eq. 18 in place of Eq. 7.

Eq. 18

$$
I=\frac{\alpha}{1-\cos \theta_{\max }} \int_{0}^{\theta_{1}}\left(\left|R_{s}\right|^{2}+\left|R_{p}\right|^{2}\right) \sin \theta d \theta
$$

Eq. 18 means that the interference of $r_{p}$ and $r_{s}$ components is neglected. In other words, this latter calculation is equivalent to our model without polarization and also to Wiegand's model with planar interfaces.

From the comparison of these three models, one can draw several conclusions. The dampening of intensity oscillations at low INA is clearly due to nonplanar interface effects. This can be explained by the faster loss of light coherence versus fringe order with object of higher curvature. However, light coherence is expected to be lost at smaller fringe orders when working at higher INA. Therefore, the nonplanar interface effects, which appear at high fringe order, are expected to be 
less important at large INA. Indeed, Wiegand et al.'s model does not significantly improve the relevance of the calculations at large INA. Finally, it also appears on Figure 6.c that polarization effects are mostly important at large INA. Obviously, an ideal model should take into account both nonplanar effects and polarization effects. However, such a model would be complex, and most importantly, it would be inadequate for the profile reconstruction of objects with unknown shape. Moreover, one can see that our calculations match already well the experimental data under all INA conditions. The good consistency for the first two to three fringe orders permits an absolute height determination of the contact zone with a precision estimated to be about $5 \mathrm{~nm}$ (Figure S1 in Supporting Information), whereas the positions of the extrema, which are correct up to five to seven fringe orders, permit us to reconstruct the shape of objects to large height (e.g., around 1500 $\mathrm{nm}$ for objects with curvature of around $10 \mu \mathrm{m})$.

3.2.4. Vesicles. We consider now the case of giant unilamellar vesicles settling on a flat substrate. These experiments serve as model for height measurements with complex objects characterized by multiple reflecting interfaces ${ }^{15,16}$ such as living cells. Figure 7 reports experimental intensity profiles and corresponding fits for DOPC vesicles on glass substrate. These experiments correspond to vesicles of radii equal to $16 \mu \mathrm{m}$ (Figure 7.a) and $11 \mu \mathrm{m}$ (Figure 7.b). For each vesicle, we report two pictures taken at two different INA values, one corresponding to the minimum accessible INA of 0.48 and the other corresponding to a larger INA value. The corresponding intensity profiles for each vesicle and each INA condition are also reported. Our model accurately fits the experimental data for up to two to three oscillation orders, with $h_{c}$ as the only fitting parameter. Note that the solution $h_{c}$ permitting us to fit the intensity profile of a given picture is not necessarily unique at low INA because the intensity is quasi-periodic with the thickness. In the case of Figure 7.a, we find for the data at INA = 0.48 two solutions with $h_{c}=90$ and $306 \mathrm{~nm}$ and for the data at INA $=1.2$ only one solution at $h_{c}=90 \mathrm{~nm}$. Hence, the measurements at two different INA conditions permit us to determine that the actual height of the vesicle is $h_{c}=90 \mathrm{~nm}$. By the same token, in the case of Figure 7.b, we find for the data at INA=0.48 two solutions with $h_{c}=15$ and $228 \mathrm{~nm}$ and for the data at INA $=0.9$ only one solution with $h_{c}=205 \mathrm{~nm}$. The actual height of the vesicle is therefore about $220 \mathrm{~nm}$. The slight difference between $h_{c}=205$ and $228 \mathrm{~nm}$ can be attributed to the dynamic height fluctuations of the vesicle ${ }^{28}$ because the pictures at different INA are not simultaneous. To our knowledge, this is the first attempt to fit RICM intensity profiles quantitatively in the case of vesicles. The remarkable agreement of the experimental and theoretical profiles even at a large INA value of 1.2 demonstrates the great accuracy of our model in describing RICM images. The fit of the profiles yields a quantitative determination of vesicle-substrate distances with a precision, according to our fitting procedure, of around \pm 5 
nm. Moreover, the use of two pictures under different INA conditions permit us to discriminate between the degenerate solutions inherent in RICM modeling.

It is interesting to underline a practical example of how the normalization procedure permits us to avoid significant errors in the determination of absolute heights with pictures taken at large INA values. Let us consider the case of Figure 7.a at high INA $=1.2$. In this example, $h_{c}$ belongs to region II of the classification of Figure 5 . The minimum of the intensity in the image corresponds to the contact zone point, and the next-order minimum is brighter than the contact zone. A simple arccosine transformation would indicate that $h_{c}=h_{1}=44 \mathrm{~nm}$ for the vesicle of Figure $7 . \mathrm{a}$ as for all vesicles belonging to region II in Figure 5. In fact, the actual distance $h_{c}$ has been found to be equal to $90 \mathrm{~nm}$ for the vesicle of Figure 7.a. This demonstrates how our model, combined with an appropriate normalization procedure, provides a significant improvement in the determination of absolute distance, especially at large INA. It is possible to estimate the difference $\Delta h_{c}$ in the absolute determination of $h_{c}$ between the simple arccosine model with three interfaces ${ }^{16}$ and our model versus the actual distances and the INA conditions (Figure 7.c). The error $\Delta h_{c}$ remains within $10 \mathrm{~nm}$ at INA $=0.48$ but can reach up to $70 \mathrm{~nm}$ at INA $=1.2$. The maximum of errors corresponds to a vesicle height $h_{c}$ equal to the height $h_{i}$ defined in Figure 5.

3.3 Objects of Unknown Shape: Height Determination and Profile Reconstruction. The previous examples with spherical objects have demonstrated the accuracy of our model in describing intensity profiles of RICM objects sitting on a flat surface. We intend here to show how the model can also be used to determine the height and reconstruct the profile of objects of unknown shape in and in the vicinity of the contact zone. Figure 8.a reports the raw intensity profiles $I(x)$ of a DOPC vesicle settling on a glass substrate and the normalized profiles $I_{N}(x)$ using the minimum and maximum intensity values available in the picture. We then calculate, using our model, the RICM intensity $I_{c}(h)$ for a planar DOPC membrane close to a flat glass substrate versus the distance $h$ between the membrane and the substrate (Figure 8.b). For a vesicle distant by a minimum distance $h_{c}$ from the substrate, the normalized intensity dependence versus the distance $I_{N c}(h)$ is deduced from $I(h)$ using the extrema of intensity corresponding to $h$ values larger than $h_{c}$. This calculation of $I_{N c}(h)$ is iterated for increasing $h_{c}$ values, starting from $h_{c}=0$, until the calculated normalized intensity and the experimental normalized intensity have the corresponding minima of intensity, at least for the lowest orders of oscillation. This is fulfilled for $h_{c}=90 \mathrm{~nm}$ and the corresponding intensity profile is reported in Figure 8.c. One can check on Figure 8.a,c, that $I_{N c}\left(h_{c}\right)=I_{N}(0)=0$ for the first minimum and $I_{N c}=I_{N} \approx 0.35$ for the second minimum. At the end of this iteration process, we 
have then determined that the minimal distance between the object and the substrate is equal to $h_{c}=90 \mathrm{~nm}$. The calculated intensity of Figure 8.c can then be used to reconstruct the shape of the object. They indeed provide correspondence between the normalized intensity and an absolute distance. Such a reconstruction is reported in Figure 8.d. For the points around the contact zone corresponding to a height $h$ smaller than the height of the first apparent maximum of intensity, a direct correspondence between the experimental intensity and the calculated values of the first branch of intensity oscillation is used. For larger heights, we instead pick up the position of the extrema and minima on the picture and attribute them to the height calculated for the corresponding extrema on the calculated curve $I_{N c}(h)$. This later process has proven to be more accurate for large heights than the application of a direct correspondence between an intensity and a corresponding height. Indeed, for high oscillation orders (i.e., large heights) the positions of the intensity extrema versus $h$ are more precisely predicted by the model than the intensities. One can see on Figure 8.d that the profile reconstructed by our method is quite similar to the profile of a sphere of diameter $16 \mu \mathrm{m}$, which is the diameter measured by phase contrast microscopy at the equator plane of the vesicle. This result is expected because the image of Figure 8 corresponds to a vesicle at iso-osmolarity between its inside and outside. This validates our shape-reconstruction process with a spherical vesicle. Now the same process can be similarly applied to a nonspherical object of unknown shape. Figure 9.a presents a RICM picture of a deflated vesicle with a noncircular contact zone surrounding noncircular Newton rings. A normalized profile $I_{N}(x)$ across the contact zone and the Newton rings is reported in Figure 9.b and the reconstruction of the shape of this profile is presented on Figure 9.c. As a comparison, the profile corresponding to a sphere of diameter of $24 \mu \mathrm{m}$ equal to the diameter measured by phase contrast at the equator plane of the vesicle is also reported on the same Figure. One can verify that the vesicle close to the substrate is flatter than the sphere, which is consistent with the idea of a floppy vesicle. A 3D representation of the contact zone can also be reconstructed by a similar process (Figure 9.d). Such reconstruction is very useful in studying the dynamics of the cell substrate contact zone topography in the early states of the cell-adhesion processes. ${ }^{29}$ Our model in this context is very valuable because it provides great improvements in the analysis of experiments performed at high INA conditions, which is typically done in RICM experiments with living cells in order to limit reflection effects from the inner organelles and increase the lateral resolution.

\section{Conclusion}

We have presented a new optical model for reflection interference contrast microscopy of immersed objects. The model permits to determine the absolute thickness of thin films with a precision of a few nanometers. Typical applications are 
the precise determination of the distance between an object (bead, vesicle, living cell) and a substrate, and the reconstruction of the 3D topography of the contact zone. The model takes into account (1) multiple reflection between a planar surface and a multi-stratified object (2) finite aperture illumination and (3), for the first time, polarization of light. We have also shown that calculated and experimental data must be carefully normalized using the intensity extrema of the same fringe order to avoid important errors in the absolute determination of small object-substrate distances, especially with images taken at high aperture illumination. The model and data normalization process have been applied successfully to filled spherical beads and to spherical giant monolamellar vesicle sitting on flat glass substrates. Experimental RICM intensity profiles of these spherical objects are well fitted up to 2-3 orders of oscillations, which insures a high precision in the determination of the absolute object-substrate distance. We have also demonstrated how the use of images taken at two different INA permits to solve the degeneracy of the solution. This extends the use of our method to distances up to $500 \mathrm{~nm}$. Our RICM data treatment is particularly interesting for dynamic RICM experiments, where high speed acquisition requires the use of high INA, and for RICM experiments with living cells where the use of large INA permits to limit multiple reflection by internal organelles. Furthermore, this new model, because it is taking into account light polarization effects, is the only one in the literature adapted for developing new substrates with low reflectance in RICM mode ${ }^{23}$. Such substrates are interesting for RICM as they yield highly contrasted images with films of nanometric thickness and the contrast enhancement of these substrates relies entirely on the reflection properties of polarized light. Finally, the model is simple enough to be usable by all RICM users even with complex systems made of multiple reflecting interfaces. This may permit its widespread use for characterization of cell/vesicle adhesion, measurement of the hydration layer thickness under a supported bilayer, or any other thickness determination in multilayer systems.

Acknowledgements: We are grateful to the society Nanolane (Le mans, France) for financial support of this work and of the PhD grant of ZHH. We also thank Laurent Limozin and Annie Viallat for fruitful discussions.

Supporting Information Available: Figure S1 and S2 report a quantitative data on the absolute precision of our model and comparisons with the simple Arccosine model for height determination and shape reconstruction. This information is available free of charge via the Internet at http://pubs.acs.org. 
List of Figures :

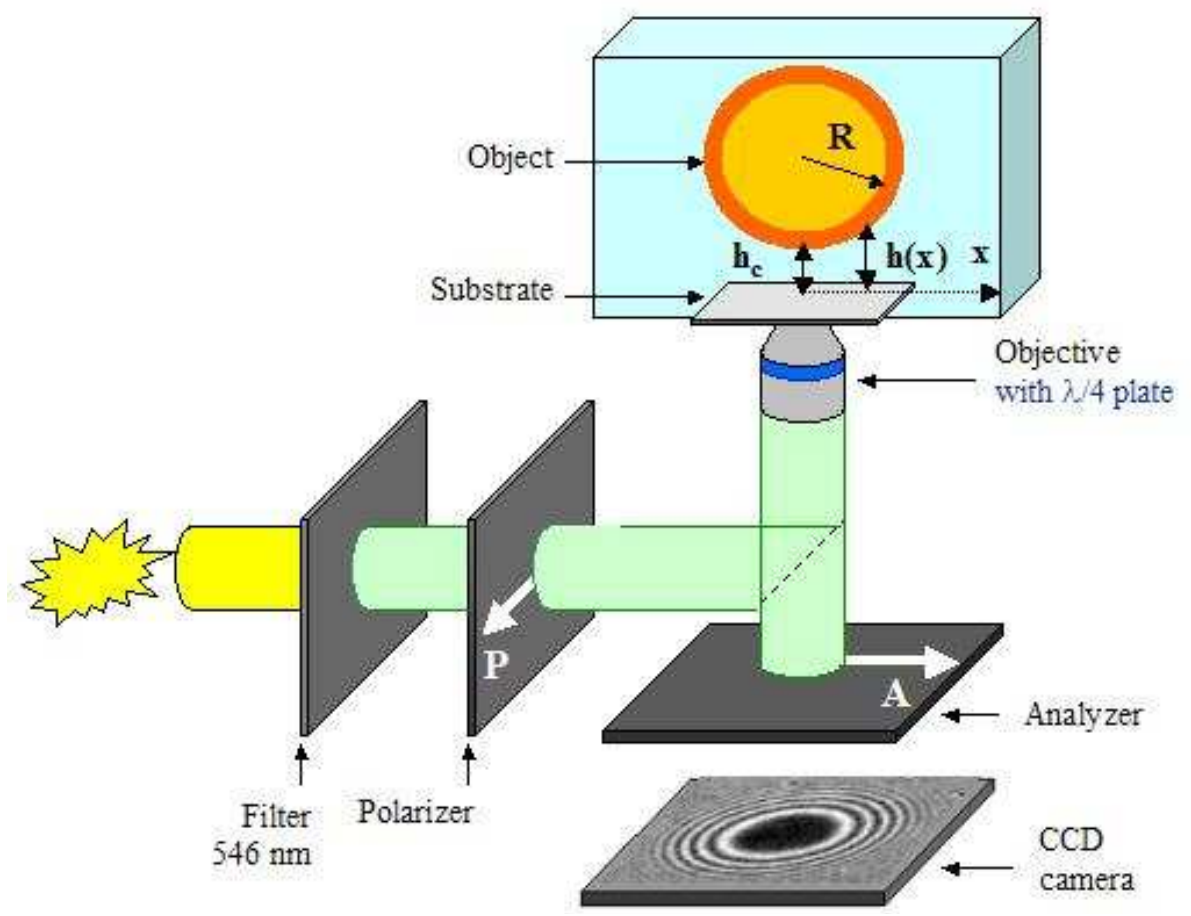

Figure 1: Schematic of a reflection contrast microscopy (RICM) set up.
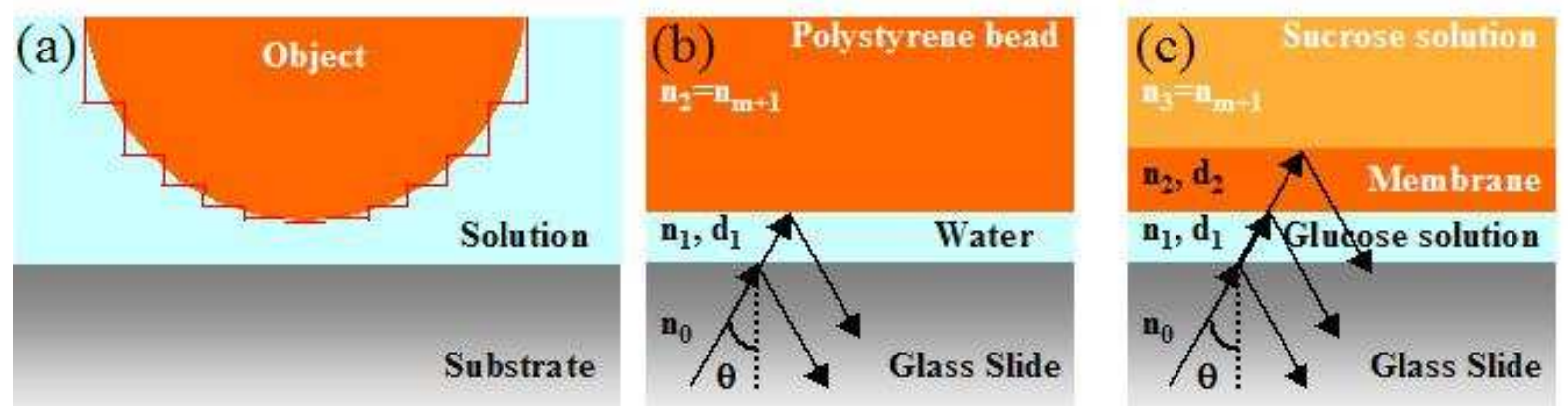

Figure 2: (a) Schematics of the RICM model of an immersed object close to a substrate. The interfaces are assumed to be locally parallel. Optical multilayer model used for (b) a spherical polystyrene bead and (c) a vesicle settled on a glass substrate. 
(a)

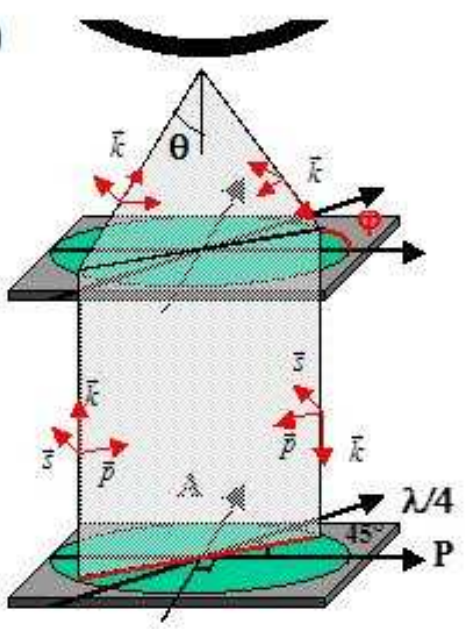

(b)

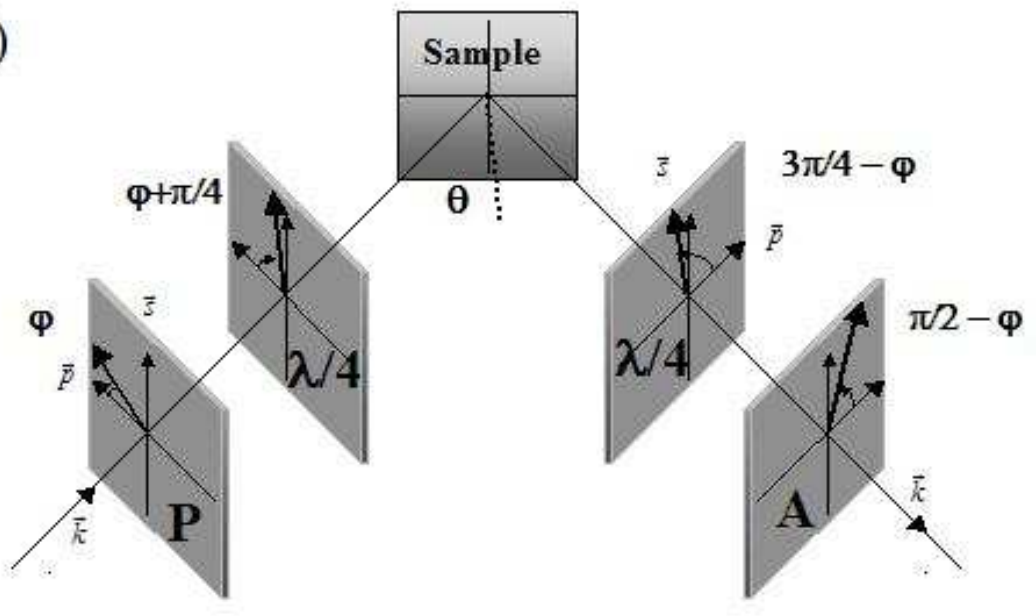

Figure 3: (a) Schematics of the illumination geometry for RICM. The $-p$ and $-s$ components are displayed for a plane of incidence of azimuth $\varphi$ with respect to the direction of the polarizer. (b) Diagram showing the ellipsometer-like arrangement under consideration for a particular beam of incidence $\theta$ and azimuth $\varphi$. All azimuths are measured positive in a counterclockwise sense looking into the beam from the direction of the - $p$ linear eigenpolarization.

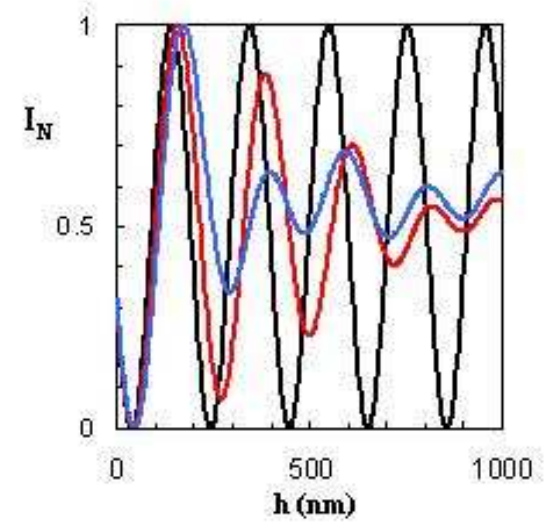

Figure 4: Calculated reflected intensity in RICM mode for a lipid membrane on a glass substrate as a function of the water film thickness $h$ separating the membrane and the substrate, for $I N A=0$ (black), INA $=0.87$ (red) and INA $=1.16$ (blue). The calculations use the refractive index $n_{0}=1.525, n_{1}=1.339, n_{2}=1.486, n_{3}=1.346$ a membrane thickness $d_{2}=5 \mathrm{~nm}$ and a wavelength $\lambda=546 \mathrm{~nm}$. 
(a)

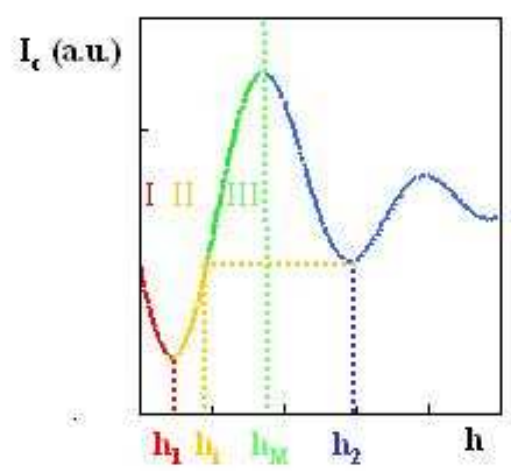

(b)

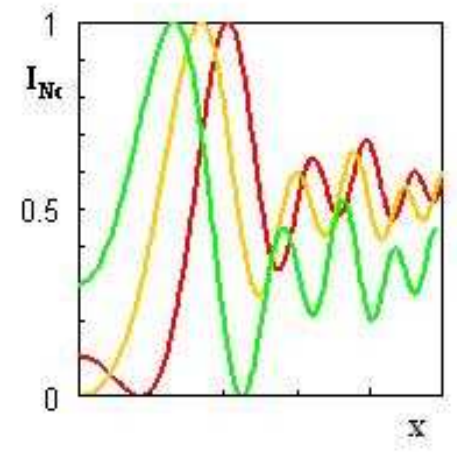

(c)

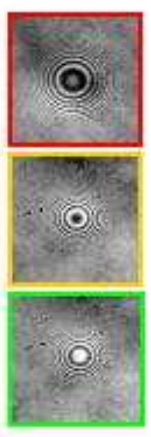

Figure 5: (a) Reflected intensity $I_{c}(h)$ calculated with eq. 7 for a lipid membrane sitting on glass vs the separating height $h$. We define three height regions based on the first intensity extrema, region I (red), region II (yellow), and region III (green). (b) Calculated normalized intensity profiles $I_{N C}(x)$ vs the radial distance $x$ for spherical vesicles sitting on glass substrates at different minimal vesicle-substrate distance $h_{c}$. The normalization uses eq. 1 and extrema $I_{\min }$ and $I_{\max }$ corresponding to $h>$ $h_{c}$. The minimal vesicle-substrate distance $h_{c}$ belonging either to Region I (solid red line), II (solid yellow line), or III (solid green line). (c) Examples of experimental RICM pictures of vesicles on glass substrates where $h_{c}$ belongs to region I (red frame), region II (yellow frame), and region III (green frame).
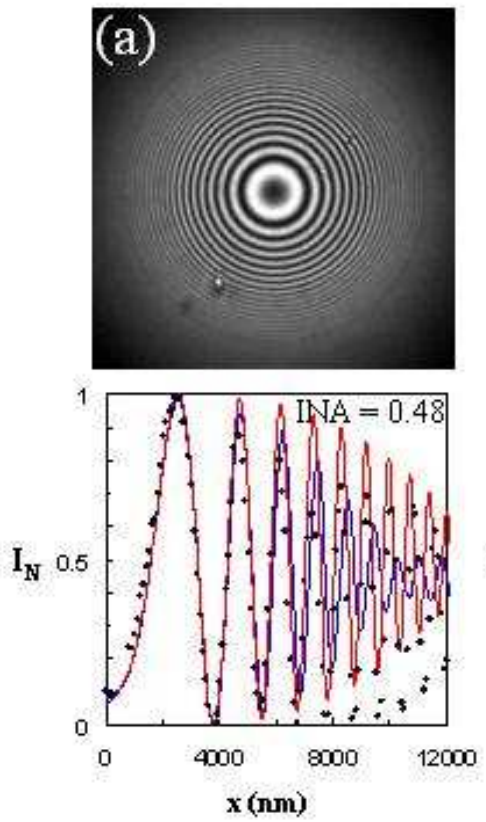
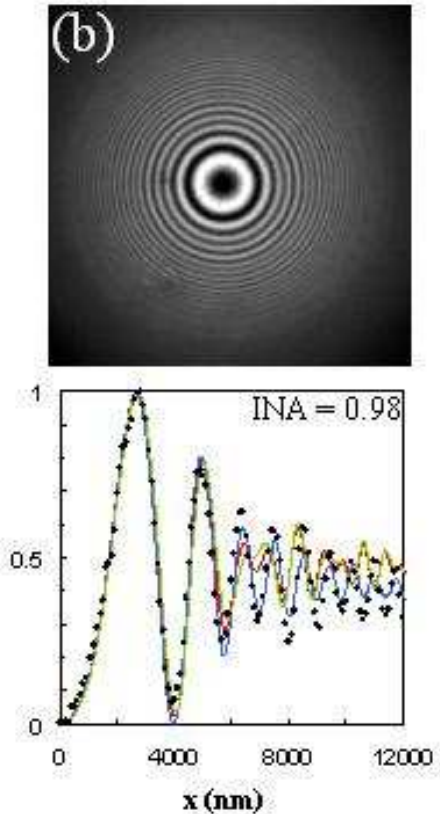
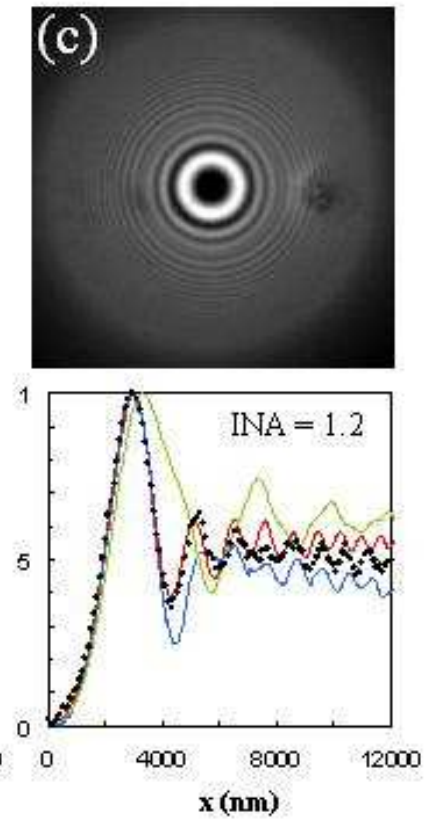

Figure 6: Effect of INA and object curvature with beads. RICM pictures for a same polystyrene bead of radius $r_{\text {bead }}=34$ $\mu \mathrm{m}$ settled on a glass substrate at illumination numerical apertures of (a) $I N A=0.48$, (b) $I N A=0.98$, and (c) $I N A=1.2$. The 
graph reports for each picture the normalized experimental intensity profiles (dots) and the calculated intensity using our model (solid red line), our model without light polarization effects or equivalently model of ref. 9 with planar interfaces (green solid line), and the model of ref 9 which takes into account non planar interfaces effects (solid blue line). The minimal separation distance $h_{c}$ between the bead and the substrate is the only fitting parameter and is taken at $h_{c}=22 \mathrm{~nm}$ for all INA values. In (a) the green and red solid lines are mingled.
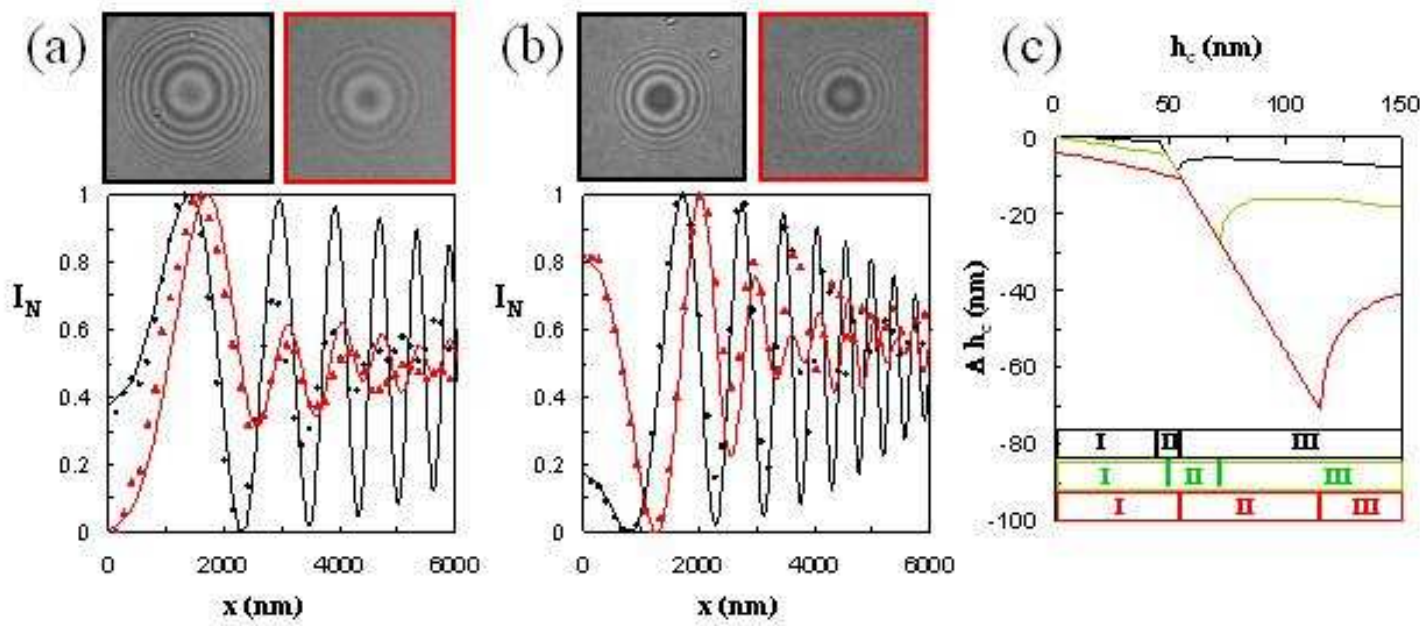

Figure 7: RICM images and normalized RICM intensity profiles for spherical vesicles of radii (a) $R=16 \mu \mathrm{m}$ and (b) $\mathrm{R}=11$ $\mu \mathrm{m}$ on a glass substrate. The color of the pictures frames and of the intensity profile indicates the INA of he experiment, (a) $I N A=0.48$ (black) and 1.2 (red), and (b) INA $=0.48$ (black) $=0.9$ (red). Symbols correspond to experimental data and solid lines are fits using eq. 7 with (a) $h_{c}=90 \mathrm{~nm}$ and (b) and $h_{c}=205$ (black) and 215 (red) nm. (c) Difference $\Delta h_{c}$ in the absolute determination of $h_{c}$ between the simple arccosine model with three interfaces (ref. 16) and our model for INA equal to 0.48 (black), 0.9 (green) and 1.2 (red). 

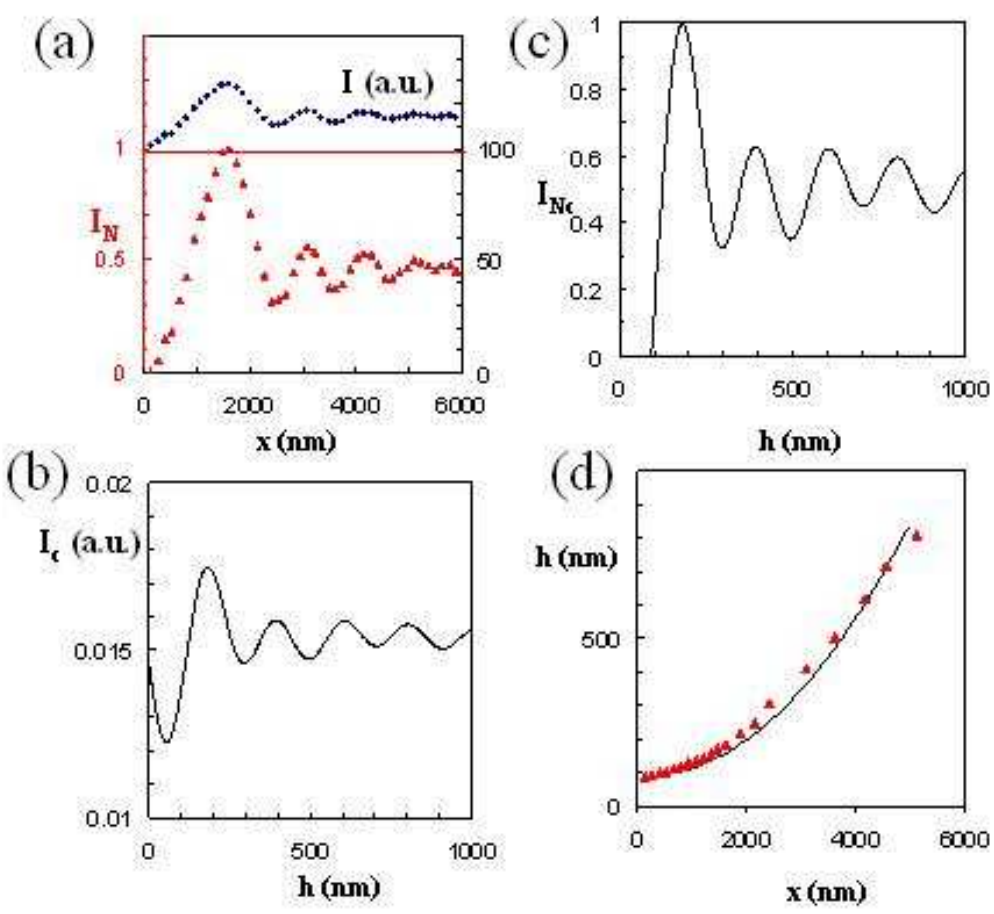

Figure 8: Shape reconstruction process. (a) Raw $I(x)$ and normalized $I_{N}(x)$ intensity profiles corresponding to the vesicle in Figure 7.a at INA $=1.2$. versus the radial distance $x$. (b) Calculated intensity $I_{c}(h)$ for a DOPC membrane on a glass substrate versus the height $h$. (c) Calculated normalized intensity $I_{N c}(h)$ calculated with a vesicle height $h_{c}=90 \mathrm{~nm}$. $h_{c}$ is determined by matching the first extrema of the curves $I_{N}(x)$ and $I_{N c}(h)$. (d) Shape profile reconstruction of the vesicle starting from the contact zone (triangle) and profile of a sphere of diameter $16 \mu \mathrm{m}$, which is the diameter of the vesicle at the equator plane measured by phase contrast microscopy (solid line). 

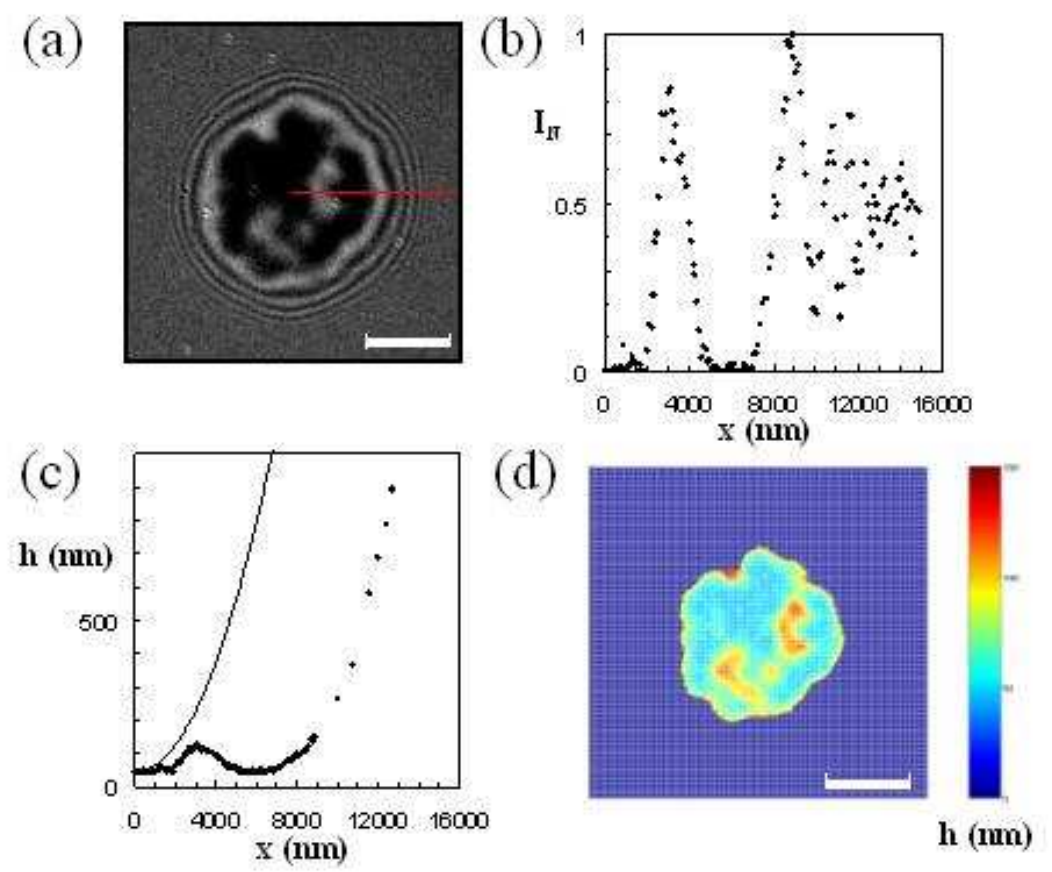

Figure 9: Shape reconstruction of a deflated vesicle on a glass substrate. (a) RICM picture taken at INA $=0.48$. (b) Normalized experimental intensity profiles $I_{N}(x)$ corresponding to the red line in panel a versus the radial distance $x$. (c) Shape profile reconstruction corresponding to the intensity profile in panel b. (d) Two-dimensional reconstructed topography of the contact zone between the vesicle and the substrate. The scale bar in panels a and $\mathrm{d}$ correspond to $4 \mu \mathrm{m}$.

\section{Supporting Information Text.}

We present additional data to document the precision of our model for absolute height determination. Figure S1 compares the fits obtained for the intensity profiles of the vesicle of Figure 7-a at INA $=0.48$ and 1.2 with different values of the fitting parameters $h_{c}$. The best fit corresponds to $h_{c}=90 \mathrm{~nm}$. It appears clearly that a deviation of $\pm 5 \mathrm{~nm}$ in $h_{c}$ yields important discrepancies between the calculations and the experimental data, especially at $\mathrm{x}=0 \mu \mathrm{m}$ at low INA $=0.48$ and at the value of the first minimum at large INA $=1.2$. This typical example permits to estimate that the precision of our model for absolute height determination is within $5 \mathrm{~nm}$. 

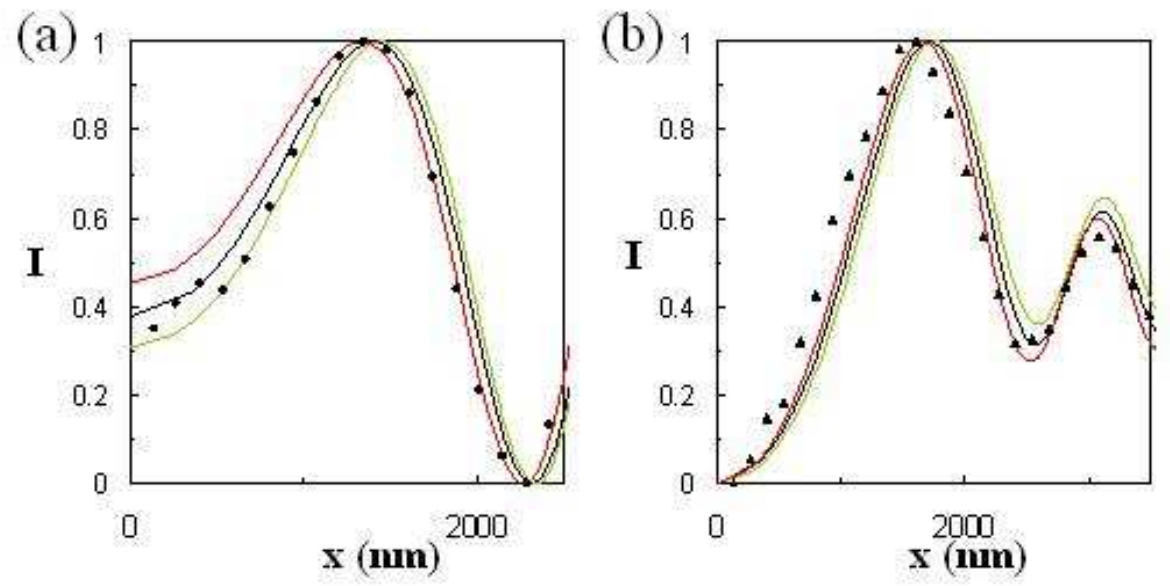

Figure S1: Intensity profiles for the vesicle of Figure 7-a model at INA $=0.48$ (a), INA = 1.2 (b): experimental data (dots) and fits obtained with our with different fitting parameters $h_{c}=90 \mathrm{~nm}$ (black), $h_{c}=85 \mathrm{~nm}$ (green), and $h_{c}=95 \mathrm{~nm}$ (red).

We show on Figure S2 how our model can be compared to the convenient Arccosine method taking into account three reflection planes (ref 16). As explained in the main text, the largest deviation between the two models appear at large INA. Figure S2 shows the profile reconstructions of the spherical vesicle of Figure 7-a at INA $=1.2$. One can see that both models lead to reconstructed profiles that are very close to the profile of a sphere. The main difference between the two models concerns the absolute distance $h_{c}$, which is emphasized in the inset of Figure S2. Our model finds $h_{c}=90 \mathrm{~nm}$ whereas the simple model yields $h_{c}=45 \mathrm{~nm}$.

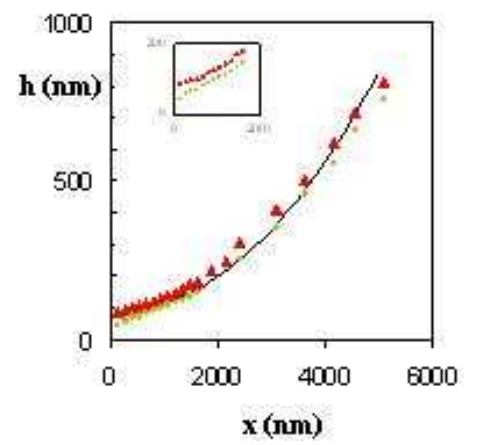

Figure S2: Shape profile reconstruction of the vesicle starting from the contact zone using our model (red triangle) and the simple Arccosine model with three interfaces (Ref. 16) for the vesicle picture of Figure 7-a at INA = 1.2. The black line corresponds to the profile of a sphere of diameter $16 \mu \mathrm{m}$, which is the diameter of the vesicle at the equator plane measured by phase contrast microscopy. 
${ }^{1}$ Nardi, J.; Feder T.; Bruinsma, R.; Sackmann, E. Europhys. Lett 1997, 38, 159-160.

${ }^{2}$ Rädler, J. O.; Feder, J. F.; Strey, H. S.; Sackmann, E. Phys. Rev. E 1995, 51, 4526-4536.

${ }^{3}$ Puech, P.-H.; Askovic, V.; De Gennes, P.-G.; Brochard-Wyart, F. Biophys. Rev. and Lett. 2006, 1, 85-95.

${ }^{4}$ Bernard, A.-L.; Guedeau-Boudeville, M.-A.; Jullien, L.; di Meglio, J.-M. Langmuir 2000, 16, 6809-6820.

${ }^{5}$ Curtis, A. S. J. Cell. Biol. 1964, 20, 199-215.

${ }^{6}$ Gingell, D.; Todd, I. Biophys. J. 1979, 26, 507-526.

${ }^{7}$ Verschueren, H. J. Cell. Sci. 1985, 75, 279-301.

${ }^{8}$ Rädler, J.; Sackmann, E. J. Phys. II France 1993, 3, 727-748.

${ }^{9}$ Wiegand, G.; Neumaier, K. R.; Sackmann, E. Applied Optics 1998, 37 (29), 6892-6905.

${ }^{10}$ Weber, I. Meth. Enzymol. 2003, 361, 34-47.

${ }^{11}$ Parthasarathy, R.; Groves, J. T. Cell Biochem. Biophys. 2004, 41, 391-413.

${ }^{12}$ Axelrod, D. J. Cell Biol. 1981, 89, 141-145.

${ }^{13}$ Gingell, D.; Todd, I.; Bailey, J. J. Cell Biol. 1985, 100 (4), 1334-1338.

${ }^{14}$ Pelham, R. J. JR; Wang, Y.-L. Proc. Natl. Acad. Sci. USA 1997, 94, 13661-13665.

${ }^{15}$ Wiegand, G.; Javorek, T.; Wegner, G.; Sackmann, E. J. Colloid. Interf. Sci. 1997, 196, 299-312.

${ }^{16}$ Limozin, L.; Sengupta, K. Biophys. J. 2007, 93, 3300-3313.

${ }^{17}$ Lorz, B. G.; Smith, A.-S.; Gege, C.; Sackmann, E. Langmuir 2007, 23 (24), 12293-12300.

${ }^{18}$ Cuvelier, D; Nassoy, P. Phys. Rev. Lett. 2004, 93, 228101.

${ }^{19}$ Smith, A.S; Lorz, B. G.; Seifert, U.; Sackmann, E. Biophys. J. 2006, 90, 1064-1080.

${ }^{20}$ Pierres, A.; Benoliel, A.-M.; Touchard, D.; Bongrand, P. Biophys. J. 2008, 94 (10), 4114-4122.

${ }^{21}$ Abkarian, M.; Lartigue, C.; Viallat, A. Phys. Rev. E. 2001, 63, 041906-17.

${ }^{22}$ Azzam, R. M. A.; Bashara, N. M. Ellipsometry and Polarized light; Elsevier science publishers B. V.: North-Holland 1987; Chapter 3.

${ }^{23}$ Ausserré, D.; Valignat, M.-P. Nano Letters 2006, 6, 1384-1388.

${ }^{24}$ Theodoly, O.; Gabriele, S.; Valignat, M.-P Opt. Express 2008, 16, 4547-58.

${ }^{25}$ Abelès, F. Ann. De Physique 1950, 5, 596-640.

${ }^{26}$ Schilling, J.; Sengupta, K.; Goennenwein, S.; Bausch, A. R.; Sackmann, E. Phys. Rev. E 2004, 69, 021901.

${ }^{27}$ Bruinsma, R.; Behrisch, A.; Sackmann, E. Phys. Rev. E 2000, 61, 4253-4267. 
${ }^{28}$ Marx, S.; Schilling, J.; Sackman, E.; Bruinsma, R. Phys. Rev. Let. 2002, 88, 138102.

${ }^{29}$ Pierres, A.; Benoliel, A.-M. ; Touchard, D. ; Bongrand, P. ; Biophys. J. 2008, 94, 4114-4122. 